\title{
Study on oxidative stress and antioxidant level in patients of acute myocardial infarction before and after regular treatment
}

\author{
Bashar T, Akhter N \\ Department of Pharmacology, Bangabandhu Sheikh Mujib Medical University, Shahbag, Dhaka. \\ Email: tahminabashar6@gmail.com
}

\begin{abstract}
In acute myocardial infarction (AMI), lack of oxygen delivery to myocardium leads to generation of reactive oxygen species (ROS) which play an important role in the pathogenesis of AMI. Endogenous anti-oxidants protect the myocardial tissues from the deleterious effect of free radical mediate injury. The study evaluates the extent of oxidative stress and antioxidant status against ROS in AMI patients and amelioration of oxidative stress after regular treatment and also assesses the association between oxidative stress and risk factors for atherosclerosis like dyslipidemia and diabetes mellitus (DM). The study was conducted on 72 AMI patients and age and sex matched 18 healthy controls. Patients were assigned to four groups, AMI without dyslipidemia or DM, with dyslipidemia, with DM and with both dyslipidemia and DM. Plasma malondialdehyde (MDA) and GSH content and vitamin E levels were determined on admission into hospital and on the $5^{\text {th }}$ day of treatment. Plasma MDA level increased significantly $(\mathrm{p}<0.001)$ and erythrocyte GSH and plasma vitamin E levels were decreased $(\mathrm{p}<0.001)$ in all the groups of patients as compared to control. On the $5^{\text {th }}$ day of regular treatment MDA level reduced $(\mathrm{p}<0.001)$ and GSH and vitamin E levels increased $(\mathrm{p}<0.001)$ in patients. The plasma MDA level was significantly higher $(\mathrm{p}<0.001)$ in patients with both dyslipidemia and DM or with only DM in comparison to patients without dyslipidemia and DM. The difference in the GSH level between patients with risk factors and without risk factors was not significant. It may be conclude that an imbalance exists between oxidant and antioxidant molecules in AMI patients which shift towards oxidative side and regular treatment restores this balance. There may be some association between oxidative stress in AMI and risk factors like dyslipidemia and diabetes mellitus.
\end{abstract}

\section{Introduction}

Acute myocardial infarction is one of the leading causes of mortality and morbidity in the world. ${ }^{1}$ AMI generally occurs following an abrupt impediment of coronary blood flow after a thrombotic occlusion of a coronary artery. AMI occurs when a coronary artery thrombus develops rapidly at a site of vascular injury caused by atherosclerosis. $^{2}$ The injury is produced or facilitated by factors such as hypertension, dyslipidemia, diabetes etc. ${ }^{3}$ Besides these factors, it has been suggested that reactive oxygen species (ROS) may play an important role in the pathogenesis of AMI. ${ }^{4}$ A decrease in the blood supply to the heart caused by thrombosis is known to induce myocardial ischemia. Mitochondrial carriers remain in a reduced state during ischemia. Following ischemia, there will be reperfusion in the myocardium where interaction between molecular oxygen and the reduced respiratory chain leads to formation of ROS. ${ }^{5,6}$ However, ROS can arise from other sources as well. ROS may have several different deleterious effects on heart. The effect of increased oxidative stress is the oxidative damage of proteins, lipids, DNA and enzymes of myocardial cells involved in energy production. These factors contribute to myocardial cell damage, extensive necrosis and cellular oedema. ${ }^{7}$ In addition, increasing ROS leads to peroxidation of lipid membranes and loss of membrane integrity, resulting in necrosis and cell death. ${ }^{8}$ Lipid peroxidation leads to generation of a variety of aldehydic products of which malandialdehyde (MDA), a stable end product, is frequently used as a marker of ROS production. ${ }^{9}$ The deleterious effect of ROS is balanced by the antioxidant action of non-enzymatic as well as enzymatic antioxidants. Vitamins and reduced glutathione (GSH) are important non-enzymatic antioxidant defenses. ${ }^{10}$ Vitamin $\mathrm{E}$ has a strong antioxidant capacity. It plays an important role in maintaining cell membrane integrity by interacting with membrane phospholipids and thereby limiting lipid peroxidation by ROS. ${ }^{11} \mathrm{GSH}$, the most important endogenous antioxidant that maintains $\mathrm{SH}$ groups 
of proteins in reduced state, detoxifies ROS and prevents ROS induced cellular damage. ${ }^{12,13}$ There is evidence that antioxidants can protect against free radical defense and may thereby inhibit thrombosis, myocardial damage and arrhythmias during AMI. In AMI oxidative stress is developed when there is excessive production of ROS, outstripping endogenous antioxidant mechanism and antioxidant status is the critical tool for assessing redox status. ${ }^{14}$

AMI is a multifactorial disease. Relative recent studies have demonstrated an association between increased oxidative stress and diabetes, hypertension, dyslipidemia, smoking etc. which are well known risk factors for atherosclerosis. ${ }^{15}$ Lipid profile has been found to be deranged in AMI patients. Increased oxidative stress and generation of ROS can result in modification of LDL to oxidized LDL that could lead to further generation of ROS. ${ }^{16}$ Under oxidative stress not only LDL, but other plasma lipids are exposed to oxidation. ${ }^{17}$ In diabetic patients, hyperglycemia increases the production of ROS by auto-oxidation of lipoproteins, proteins and glucose. ${ }^{18}$ Diabetes mellitus reduces the availability of nitric oxide (NO) ${ }^{19}$ which maintain antioxidative capacity of endothelial cells. $^{20}$ The present study is therefore, designed to evaluate the extent of oxidative stress and antioxidant status against ROS in AMI patients before and after regular treatment by measuring plasma MDA, reduced GSH and vitamin E levels. In addition the study will also evaluate an association between oxidative stress and risk factors for atherosclerosis like dyslipidemia and diabetes mellitus.

\section{Materials and Methods}

This prospective type of observational study was conducted in the department of Pharmacology, Bangabandhu Sheikh Mujib Medical University (BSMMU) and National Institute of Cardiovascular Diseases (NICVD), Dhaka from January to December 2010.

Study population: The study population consisted of 72 AMI patients (age: 40-70 yrs) who were admitted in Coronary Care Unit (CCU) of NICVD. The diagnosis of AMI was done by cardiologists based on clinical criteria: prolonged ischemic chest pain, characteristic ECG changes (ST elevation of $2 \mathrm{~mm}$ or more in at least 2 contagious leads) and elevated creatine kinase isoenzyme MB (CK-MB) and troponin $\mathrm{T}$ within 8 hours after the onset of pain. ${ }^{21}$ Some general characteristics of patients were noted e.g. hypertension, dyslipidemia, DM etc. Hypertension was defined as a diastolic blood pressure $\geq 90 \mathrm{~mm}$ of $\mathrm{Hg}$ and systolic blood pressure $\geq 140 \mathrm{~mm}$ of $\mathrm{Hg}$ or self reported use of antihypertensive drugs. The subjects who had total cholesterol level of $<200 \mathrm{mg} / \mathrm{dL}$, triglycerides $<150 \mathrm{mg} / \mathrm{dL}$, LDL-C <160mg/dL and HDL-C >35 $\mathrm{mg} / \mathrm{dL}$ were defined as normolipidemic patients, patients treated with insulin or oral hypoglycemic agents were considered as diabetic. Patients taking anti-oxidant supplements for last 3 months, lipid lowering agents for last 6 months, hepatic or renal insufficiency, pregnant or lactating mother or who did not voluntarily agreed to participate were excluded from the study. Age and sex matched 18 healthy subjects with normal blood pressure, free from diabetes, dyslipidemia or any other chronic ailments were included as control group. Control group was recruited from Matuail Health Complex who attended in the out patient department for health check up.

The whole study population was grouped as follows:

Group I or control - 18 healthy subjects

Group II or study - 72 AMI patients

Sub-divided as follows:

Group IIa - 18 AMI patients without diabetes mellitus or dyslipidemia

Group IIb - 18 AMI patients with dyslipidemia

Group IIc - 18 AMI patients with diabetes mellitus

Group IId - 18 AMI patients with both diabetes mellitus and dyslipidemia

All the patients received conventional therapy for AMI which included Inj. streptokinase (2.5 million unit) - $50 \mathrm{ml} \mathrm{I/V} \mathrm{@} 50 \mathrm{ml} /$ hour stat; Inj. morphine (10 mg) and Inj. prochlorperazine (5mg); Tab. clopidogrel (75mg), Tab. atrovastatin (20mg). Hypertensive, diabetic, and dyslipidemic patients were treated accordingly.

Prior to study, informed written consent was taken from patients and subjects participated prior to the study. The study protocol was approved by the Ethical Review Committee of the Bangabandhu Sheikh Mujib Medical University, Dhaka, Bangladesh.

Blood samples of patients were collected twice, once on admission into the hospital before starting treatment and thence on the 5th day of treatment. In controls blood sample was taken only for once after overnight fasting.

Blood collection and erythrocyte lysate preparation: With all aseptic precautions $5 \mathrm{ml}$ blood was collected in $\mathrm{K}_{3}$ EDTA containing test tube. Plasma was separated by ultra centrifugation (1370 $\times \mathrm{g}$ for 10 minutes) and stored at labeled eppendorf. The packed cells were washed thrice with cold $0.9 \%$ saline by centrifugation. They were suspended in five volumes of deionized water at $4^{\circ} \mathrm{C}$ for 24 hours to lyse RBCs then centrifuged them to collect RBCs lysate. Finally all samples were stored at $-10^{\circ} \mathrm{C}$. 
Bio-chemical Methods: Lipid peroxidation was estimated by measurement of thiobarbituric acid reactive substances (TBARS) in plasma by the method of Yagi. ${ }^{22}$

Reduced glutathione (GSH) in RBC was determined by the method of Ellman. ${ }^{23}$

Plasma vitamin $E$ was measured by the method of Baker et al. ${ }^{24}$

Plasma glucose and lipid profiles (total cholesterol, triglycerides, LDL-C, HDL-C) were analyzed enzymatically using commercial kits.

All the chemicals and enzymatical kits were purchased from Sigma and Human, Germany.

Statistical analysis: Statistical analysis was done by SPSS software (SPSS 15.0). The quantitative variables were expressed as mean \pm SD. ANOVA followed by Bonferroni t test was done to measure level of significance for statistical analysis. $P$ value $<.05$ was considered to indicate statistical significance.

\section{Results}

Incidence of hypertension (83\%) was more amongst patients with both DM and dyslipidemia. Blood glucose level of AMI patients with DM or dyslipidemia or with both DM and dyslipidemia was significantly higher $(\mathrm{p}<0.001)$ than control (Table I). Blood glucose level was also higher $(\mathrm{p}<0.01)$ in AMI patients without DM or dyslipidemia than control. Plasma lipids showed a higher concentration $(p<0.001)$ of total cholesterol (TC), triglyceride (TG) and low density lipoproteins (LDL-C) in patients with dyslipidemia and with both dyslipidemia and DM. LDL-C level was also high $(\mathrm{p}<0.001)$ in AMI with DM. Total cholesterol level was high $(\mathrm{p}<0.05)$ in AMI patients without DM or dyslipidemia as compared to control. Total cholesterol level was significantly higher $(\mathrm{p}<0.01)$ in AMI patients with DM. The level of LDL-C was also higher $(p<0.01)$ in AMI patients without dyslipidemia or DM as compared to controls. The difference in TG concentration in
AMI patients without DM or dyslipidemia or with DM as compared to controls was not significant. On the other hand the level of HDL-C was lower $(p<0.001)$ in patients with dyslipidemia and with both DM and dyslipidemia when compared to healthy control subjects. The change in HDL-C concentration was not significant in patients without DM or dyslipidemia and in patients with DM when compared to controls.

Table II illustrates the level of lipid peroxidation and antioxidant status in patients and control subjects. Lipid peroxidative marker, MDA, was significantly higher $(p<0.001)$ in all the groups of patients as compared to control. The activity of erythrocyte antioxidant GSH and plasma concentration of vitamin $\mathrm{E}$ levels were lower $(p<0.001)$ in all the groups of patients as compared to normal subjects. Significantly higher $(p<0.001)$ plasma MDA level was observed in group IIc or IId versus group IIa. There was no significant change ( $>0.05)$ in erythrocyte GSH level when group IIb or IIc or IId was compared with group IIa. Vitamin E level was significantly higher in group IIc or IId than group IIa.

Table III illustrates the level of lipid peroxidation and antioxidant status before starting regular treatment and on the $5^{\text {th }}$ day of treatment in all patients and control subjects. Before starting regular treatment plasma MDA level was significantly higher $(\mathrm{p}<0.001)$ and erythrocyte GSH and plasma vitamin $\mathrm{E}$ levels were significantly lower $(p<0.001)$ in patients when compared to controls. After regular treatment, on the $5^{\text {th }}$ day, plasma concentration of MDA reduced significantly $(p<0.001)$ in patients. However, MDA level did not return to the normal value and its difference from control value was significant $(p<0.05)$. On the $5^{\text {th }}$ day of regular treatment non-enzymatic markers of antioxidants: erythrocyte GSH and plasma vitamin E, levels increased significantly $(p<0.001)$ in patients as compared to before starting regular treatment, but the GSH and vitamin E levels did not reach the control value.

Table I: Blood glucose level and plasma lipid profile in AMI patients and control

\begin{tabular}{|c|c|c|c|c|c|}
\hline \multirow{3}{*}{ Parameters } & \multirow[t]{2}{*}{ Control } & \multicolumn{4}{|c|}{ AMI Patients (72) } \\
\hline & & $\begin{array}{l}\text { AMI without DM or } \\
\text { Dyslipidemia }\end{array}$ & $\begin{array}{c}\text { AMI with } \\
\text { Dyslipidemia }\end{array}$ & AMI with DM & $\begin{array}{l}\text { AMI with both DM } \\
\text { and Dyslipidemia }\end{array}$ \\
\hline & $\begin{array}{l}\text { Group I } \\
(n=18)\end{array}$ & $\begin{array}{l}\text { Group IIa } \\
(n=18)\end{array}$ & $\begin{array}{l}\text { Group IIb } \\
\quad(=18)\end{array}$ & $\begin{array}{l}\text { Group IIc } \\
\quad(n=18)\end{array}$ & $\begin{array}{l}\text { Group IId } \\
\quad(n=18)\end{array}$ \\
\hline Blood Glucose (70-110 mg/dl) & $89 \pm 8.40$ & $97 \pm 2.82^{* *}$ & $104 \pm 3.79^{* * *}$ & $132 \pm 9.11^{* * * *}$ & $140 \pm 8.19^{* * * *}$ \\
\hline Total Cholesterol $(150-200 \mathrm{mg} / \mathrm{dl})$ & $162 \pm 11.23$ & $172 \pm 13.46^{*}$ & $221 \pm 17.41^{* * * *}$ & $179 \pm 18.42^{* *}$ & $238 \pm 19.17^{* * *}$ \\
\hline Triglyceride (50-150 mg/dl) & $137 \pm 21.23$ & $143 \pm 23.10^{\mathrm{NS}}$ & $177 \pm 21.26^{* * * *}$ & $141 \pm 13.43^{\mathrm{NS}}$ & $174 \pm 11.03^{* * *}$ \\
\hline LDL-C (100-160 mg/dl) & $92 \pm 9.11$ & $100 \pm 5.07^{* *}$ & $155 \pm 8.32^{* * *}$ & $110 \pm 9.72^{* * *}$ & $173 \pm 12.08^{* * *}$ \\
\hline HDL-C (40-55 mg/dl) & $43 \pm 5.09$ & $43 \pm 4.13^{\mathrm{NS}}$ & $31 \pm 4.32^{* * *}$ & $41 \pm 5.19^{\mathrm{NS}}$ & $30 \pm 6.01^{* * *}$ \\
\hline
\end{tabular}

Values were expressed as mean $\pm \mathrm{SD}$; "Unpaired t test" was done between study groups and control group Group IIa/ IIb/ IIc/ IId (study groups) compared to Group I (control)

$* * *=$ significant at $\mathrm{p}<0.001 ; * *=$ significant at $\mathrm{p}<0.01 ; *=$ significant at $\mathrm{p}<0.05 ; \mathrm{ns}=$ not significant at $\mathrm{p}>0.05$ 
Table II: Plasma MDA, plasma vitamin E and erythrocyte GSH levels in AMI patients and control

\begin{tabular}{|c|c|c|c|c|c|}
\hline \multirow[b]{2}{*}{ Parameters } & \multirow{2}{*}{$\begin{array}{l}\text { Control } \\
\text { Group I } \\
(n=18)\end{array}$} & \multicolumn{4}{|c|}{ AMI Patients (72) } \\
\hline & & $\begin{array}{c}\text { AMI without DM } \\
\text { or Dyslipidemia } \\
\text { Group IIa } \\
(n=18)\end{array}$ & $\begin{array}{l}\text { AMI with } \\
\text { Dyslipidemia } \\
\text { Group IIb } \\
(n=18)\end{array}$ & $\begin{array}{l}\text { AMI with DM } \\
\text { Group IIc } \\
(n=18)\end{array}$ & $\begin{array}{c}\text { AMI with both DM } \\
\text { and Dyslipidemia } \\
\text { Group IId } \\
(n=18)\end{array}$ \\
\hline Plasma MDA $(\mu \mathrm{mol} / \mathrm{l})$ & $2.51 \pm 0.42$ & $3.79 \pm 0.96^{\mathrm{a}^{* * *}}$ & $4.47 \pm 0.99^{\mathrm{a}^{* * *} \mathrm{bns}}$ & $4.94 \pm 0.79^{\mathrm{a}^{\mathrm{a} * * * \mathrm{c}^{* * * *}}}$ & $5.85 \pm 0.79^{\mathrm{a}^{\mathrm{a} * * *} \mathrm{~d}^{* \ldots * *}}$ \\
\hline Plasma vitamin E (mg/l) & $8.26 \pm 0.65$ & $4.89 \pm 0.78^{\mathrm{a}^{* * *}}$ & $5.38 \pm 0.90^{\mathrm{a}^{* * *} \text { bns }}$ & $5.63 \pm 0.64^{\mathrm{a}^{* * * *} \mathrm{c}^{*}}$ & $5.83 \pm 0.78^{\mathrm{a}^{* * *} \mathrm{~d}^{* *}}$ \\
\hline Erythrocyte GSH (mg/gm of $\mathrm{Hb})$ & $1.31 \pm 0.11$ & $0.91 \pm 0.09^{\mathrm{a}^{* * *}}$ & $0.93 \pm 0.07^{\mathrm{a}^{* * *} \text { bns }}$ & $0.92 \pm 0.05^{\mathrm{a}^{* * *} \mathrm{cns}}$ & $0.96 \pm 0.04^{\mathrm{a}^{* * *} \mathrm{dns}}$ \\
\hline
\end{tabular}

Values were expressed as mean $\pm \mathrm{SD}$; ANOVA followed by Bonferroni t test was done to measure level of significance

a Group IIa/ IIb/ IIc/ IId (study groups) as compared to Group I (control) b Group IIb as compared to Group IIa

c Group IIc as compared to Group IIa d Group IId as compared to Group IIa

$* * *=$ significant at $\mathrm{p}<0.001 ; * *=$ significant at $\mathrm{p}<0.01 ; *=$ significant at $\mathrm{p}<0.05 ; \mathrm{ns}=$ not significant at $\mathrm{p}>0.05$

Table III Plasma MDA, plasma vitamin E and erythrocyte GSH levels before and after treatment in AMI patients and control

\begin{tabular}{lccc}
\hline & & \multicolumn{2}{c}{ AMI Patients $n=72$} \\
\cline { 3 - 4 } Parameters & $\begin{array}{c}\text { Control } \\
n=18\end{array}$ & $\begin{array}{c}\text { Before } \\
\text { Treatment } \\
\text { At baseline) }\end{array}$ & $\begin{array}{l}\text { On the } 5^{\text {th }} \text { day } \\
\text { of Treatment }\end{array}$ \\
\hline Plasma MDA $(\mu \mathrm{mol} / \mathrm{l})$ & $2.51 \pm 0.42$ & $4.76 \pm 2.72^{\mathrm{a}^{* * * *}}$ & $2.78 \pm 2.48^{\mathrm{b}^{* * *}}$ \\
Plasma vitamin E $(\mathrm{mg} / \mathrm{l})$ & $8.26 \pm 0.65$ & $5.43 \pm 1.93^{\mathrm{a}^{* * * *}}$ & $7.68 \pm 0.91^{\mathrm{b}^{* * *}}$ \\
$\begin{array}{l}\text { Erythrocyte GSH } \\
(\mathrm{mg} / \mathrm{gm} \text { of } \mathrm{Hb})\end{array}$ & $1.31 \pm 0.11$ & $0.93 \pm 0.09^{\mathrm{a}^{* * * *}}$ & $1.17 \pm 0.29^{\mathrm{b}^{* * * *}}$ \\
\hline
\end{tabular}

Values were expressed as mean $\pm \mathrm{SD}$;

ANOVA followed by Bonferroni $t$ test was done to measure level of significance

a before treatment group compared to control group

$\mathrm{b}$ after treatment group compared to before treatment group

$* * *=$ significant at $\mathrm{p}<0.001 ; * *=$ significant at $\mathrm{p}<0.01$

$*=$ significant at $\mathrm{p}<0.05 ; \quad \mathrm{ns}=$ not significant at $\mathrm{p}>0.05$

\section{Discussion}

A decrease in blood supply to the heart due to atherosclerosis, thrombosis or coronary artery spasm is known to induce myocardial ischemia. ${ }^{2}$ Reperfusion of ischemic myocardium may restore oxygen supply. However sudden massive increase in oxygen supply causes a burst of oxygen consumption with consequent generation of reactive oxygen species (ROS). Excess production of ROS causes inhibition of antioxidant activity, ${ }^{12,25}$ which may cause modification of cellular lipids, proteins and DNA of myocardium and result in severe myocardial damage. ${ }^{7}$ In the present study lipid peroxidation product, MDA was significantly increased $(p<0.001)$ in patients as compared to control which is indicative of elevated oxidative stress in patients. Our results are consistent with other researchers findings where they showed significant increase in the lipid peroxidation products in blood of AMI patients following an acute event. ${ }^{2,26-27}$ The same result was found by Senthi et al, ${ }^{28}$ in patients with cardiogenic shock complicating AMI. Increased lipid peroxidation is thought to be a consequence of oxidative stress which occurs when the dynamic balance between pro-oxidant and antioxidant mechanism is impaired. Lipid peroxidation may alter intrinsic membrane properties due to physicochemical changes of oxidized lipids or cause polymerization of membrane components. ${ }^{29}$ The increased level of MDA suggests that lipid damage observed in our study may be a result of the AMI pathology itself.

Antioxidants constitute the foremost defense system that limit the toxicity associated with free radicals. A reason for increased lipid peroxidation of patients with AMI may be a poor enzymatic and non enzymatic antioxidant defense system. Reduced glutathione (GSH) is one of the important endogenous non-enzymatic antioxidants. It provides sulphdryl (SH) group for direct scavenging reactions. GSH acts both as substrate in the scavenging reaction catalyzed glutathione peroxidase (GPx) and as a scavenger of peroxyl radicals. ${ }^{30}$ Our data showed that GSH level was significantly lowered in patients as compared to control and a negative correlation was observed between GSH and MDA levels in patients ( $\mathrm{r}=$ -0.515 ). The finding of the study is in accordance with a number of studies. Erythrocyte GSH level has been found to be lowered significantly in AMI patients as compared to control. ${ }^{31,32}$ A negative correlation between GSH and MDA has been shown in both diabetic and non diabetic AMI patients. ${ }^{31}$ The decreased level of GSH and negative correlation of GSH and MDA content in our study implies that glutathione system, which is the important protective system against oxidative damage, is severely impaired in AMI patients. In our study, we found significantly low level of plasma vitamin $E$ level in patients compared with control. This indicates severe damage to antioxidant system which is unable to combat oxidative stress. Vitamin $\mathrm{E}$ is an important membrane constituent of cardiac muscle which haults lipid peroxidation by trapping the peroxyl radical and stabilizes lipid bilayer of cell membranes, where it interacts with phospholipases to reduce membrane rearrangements. ${ }^{25,33}$ It may provide protection against ischaemic myocardial damage by 
eliminating prooxidants and scavenging free radicals. ${ }^{34}$ The low vitamin $\mathrm{E}$ level found in our study is in accordance with other studies., $20,31,32$ Some studies showed that total antioxidant capacity (TAC) has been decreased in AMI patients. ${ }^{16,28}$ Decrease in the levels of GSH and vitamin $\mathrm{E}$ in our study may be due to increased turn over for preventing oxidative damage in these patients, suggesting an increased defense against oxidative damage in AMI patients.

In this study, on the $5^{\text {th }}$ day of regular treatment, we found significant decrease in plasma MDA level $(\mathrm{p}<0.001)$ in patients, However the level did not reach to control value. We also found significant increase $(\mathrm{p}<0.001)$ in erythrocyte GSH and plasma vitamin $\mathrm{E}$ level in patients on the $5^{\text {th }}$ day of regular treatment. LoPresti et $\mathrm{al}^{26}$ found significant reduction of lipid peroxidation and NO metabolite concentration and increase of total antioxidant status (TAS) in young AMI patients after 12 months in comparison with initial stage. However each of parameters remained statistically different in comparison to normal controls. In our study on the $5^{\text {th }}$ day of treatment we found significant difference of MDA level as compared to control but the difference of erythrocyte GSH and plasma vitamin $\mathrm{E}$ level was not significant in comparison to control. It may be that TAC takes longer period to be restored.

Numbers of risk factors are associated with the pathogenesis of AMI. Increase in the concentration of plasma lipids is frequently observed in patients with AMI which contributes to the development of vascular disease. Cholesterol has been considered to be an important factor in the development of atherosclerosis. HDL-C provides protection against it by reverse transport of cholesterol. In our study, we found significant elevation of cholesterol and LDL-C levels in AMI patients with dyslipidemia or both dyslipidemia and DM as compared to control. There was significant decrease in HDL-C in the same group of patients. In patients with only DM, LDL-C level increased significantly. We found that the rate of lipid peroxidation was significantly high in AMI patients with or without risk factors as compared to control. MDA level was significantly higher in patients with DM or both dyslipidemia and DM as compared to patients with no risk factors. No significant difference was observed in GSH level between patients with and without risk factors. Vitamin E level was significantly higher in patients with risk factors than patients without risk factors. Regarding antioxidant status we got unexpected results which could be due to smaller sample size. We could not correlate our findings with other studies since no such study with specific risk factor has been done. However LoPresti et $\mathrm{al}^{26}$ found no significant differences in thio barbituric acid reactive substances (TBARS) and TAS among the subgroups of AMI patients with risk factors like family history, smoking, hypercholesterolemia, DM, essential hypertension. In our study we found increased plasma levels of MDA in all the subgroups of AMI patients with specific risk factor. It is predicted that oxidative stress could be higher among the patients with risk factors than the patients risk having no factors.

The findings of our study suggest that in AMI patients oxidative stress occurs as a result of an imbalance between oxidants and antioxidants. The magnitude of imbalance is greater in patients with only DM or both dyslipidemia and DM. Amelioration of oxidative stress after regular treatment suggests that such an imbalance between oxidants and antioxidants may be involved in the pathogenesis of AMI.

\section{Reference}

1. Ojha SK, Nandave M, Arora S, Narang R, Dinda AK, Arya DS. Chronic administration of Tribulus terrestris Linn. Extract improves cardiac function and attenuates myocardial infarction in rats. Int J Pharmacol 2008; 4: 110.

2. Bagatini MD, Martins CC, Battisti V, Gasparetto D, da Rosa CS, Spanevello RM, Ahmed M, Schmatz R, Schetinger MR, Morsch VM. Oxidative stress versus antioxidant defenses in patients with acute myocardial infarction. Heart Vessels 2011; 26: 55-63.

3. Kasap S, Gonenc A, Sener DE, Hisar I. Serum cardiac markers in patients with acute myocardial infarction: Oxidative stress, C-Reactive protein and N-terminal probrain natriuretic peptide. J Clin Biochem Nutr 2007; 41: 50-57.

4. Loeper J, Goy J, Rozenstajin L, Bedu O, Moisson P. Lipid peroxidation and protective enzymes during myocardial infarction. Clin Chim Acta 1991;15:119-25.

5. Espat NJ and Nelton WS. Oxygen free radicals, oxidative stress, and antioxidants in critical illness. Support Line 2000; 22: 11-20.

6. Kasparová S, Brezová V, Valko M, Horecký J, Mlynárik V, Liptaj T, Vancová O, Ulicná O, Dobrota D. Study of the oxidative stress in a rat model of chronic brain hypoperfusion. Neurochem Int 2005; 46: 601-11.

7. Giordano FJ. Oxygen, oxidative stress, hypoxia, and heart failure. J Clin Invest. 2005; 115: 500-08.

8. Lazzarino G, Raatikainen P, Nuutinen M, Nissinen J, Tavazzi B, Di Pierro D, Giardina B, Peuhkurinen K. Myocardial release of malondialdehyde and purine compounds during coronary bypass surgery. Circulation 1994; 90: 291-97.

9. Aznar J, Santos MT, Valles J, Sala J. Serum malondialdehyde-like material (MDA-LM) in acute myocardial infarction. J Clin Pathol 1983; 36: 712-15. 
10. Muzáková V, Kandár R, Vojtísek P, Skalický J, Vanková R, Cegan A, Cervinková Z. Antioxidant vitamin levels and glutathione peroxidase activity during ischemia/reperfusion in myocardial infarction. Physiol Res 2001; 50: 389-96.

11. Canbaz S, Duran E, Ege T, Sunar H, Cikirikcioglu M, Acipayam M. The effect of intracoronary administration of vitamin $\mathrm{E}$ on myocardial ischemiareperfusion injury during coronary artery surgery. Thorac Cardiovasc Surg 2003; 51: 57-61.

12. Cross CE, Halliwell B, Borish ET, Pryor WA, Ames BN, Saul RL, McCord JM, Harman D. Oxygen radicals and human disease. Ann Intern Med 1987; 107: 52645 .

13. Shimizu H, Kiyohara Y, Kato I, Kitazono T, Tanizaki Y, Kubo M, Ueno H, Ibayashi S, Fujishima M, Iida M. Relationship between plasma glutathione levels and cardiovascular disease in a defined population: the Hisayama study. Stroke 2004; 35: 2072-77.

14. Ghiselli A, Serafini M, Natella F, Scaccini C. Total antioxidant capacity as a tool assesses redox status: critical view and experimental data. Free Radic Biol Med 2000; 29: 1106-14.

15. Shaikh AK and Suryakar AN. Oxidative stress and antioxidant status before and after supplementation of A-Z anti-oxidant tablets in coronary artery disease. Biomed Res 2009; 20: 136-40.

16. Chen $\mathrm{J}$ and Mehta JL. Role of Oxidative stress in Coronary Heart disease. Indian Heart J 2004; 56: 163 73.

17. Kumar A, Sivakanesan R, Singh S. Oxidative stress, endogenous antioxidants and ischemia-modified albumin in normolipidemic acute myocardial infarction patients. J Health Sci 2008; 54: 482-87.

18. Hunt JV, Smith CC, Wolff SP. Autoxidative glycosylation and possible involvement of peroxides and free radicals in LDL modification by glucose. Diabetes 1990; 39: 1420-24.

19. Potenza MA, Gagliardi S, Nacci C, Carratu MR, Montagnani M. Endotheli Dysfunction in Diabetes: From Mechanisms to Therapeutic Targets. Curr Med Chem 2009; 16: 94-112.

20. Vanhoutte PM. Endothelial dysfunction and vascular pathology. Bull Mem Acad R Med Belg 2006; 161: 529-36.

21. Thygesen K, Alpert JS, White HD. Universal definition of myocardial infarction. Circulation 2007; 116: 263453.
22. Yagi K. Lipid peroxides and human diseases. Chem Phys Lipids 1987; 45: 337-51.

23. Ellman GL.Tissue sulfhydryl groups. Arch Biochem Biophys 1959; 82: 70-77.

24. Baker H and Frank O. Determination of serum $\alpha-$ tocoperol. In: Gokenlock AH, MeMurray JR, Mehauchian DM, eds. Varleys Practical Clinical Biochemistry. London. 1968: 902-03.

25. Frei B, Stocker R, Ames BN. Antioxidant defenses and lipid peroxidation in human blood plasma. Proc Natl Acad Sci 1988; 85: 9748-52.

26. LoPresti R, Catania A, D'Amico T, Montana M, Caruso M, Caimi G. Oxidative stress in young subjects with acute myocardial infarction:Evaluation at the initial stage and after 12 months. Clin Appl Thromb Hemost 2008; 14: 421-26.

27. Aparna P, Betigeri AM, Pasupathi P. Homocystine and oxidative stress markers and inflammation in patients with coronary artery diseases. Int J Biol Med Res 2010; 1: $125-29$.

28. Senthil S, Veerappan RM, Ramakrishna Rao M, Pugalendi KV. Oxidative stress and antioxidants in patients with cardiogenic shock complicating acute myocardial infarction. Clin Chim Acta 2004; 348: 131-37.

29. Esterbauer H, Shauer RJ, Zollner H. Chemistry and biochemistry of 4-hydroxynonenal malonaldehyde and related aldehydes. Free Radic Biol Med 1991; 11: $81-128$.

30. Myers ML, Bolli R, Lekich RF, Hartley CJ, Roberts R. Enhancement of recovery of myocardial function by oxygen free radical scavengers after reversible regional ischemia. Circulation 1985; 72: 915-21.

31. Patil N, Chavan V, Karnik ND. Antioxidant status in patients with acute myocardial infarction. Ind $\mathrm{J}$ Clin Biochem 2007; 22: 45-51.

32. Deepa M , Pasupathi P, Vidhya Sankar KB, Rani P, Satis Kumar SP. Free radicals and antioxidant status in acute myocardial infarction patients with and without diabetes mellitus. Bangladesh Med Res Counc Bull 2009; 35: 95-100.

33. Evans $P$ and Halliwell B. Micronutrients: oxidant/antioxidant status. Br J Nutr 2001; 85: 67-74.

34. Bandyopadhyay D, Chattopadhyay A, Ghosh G, Datta AG. Oxidative stress-induced ischemic heart disease: protection by antioxidants. Curr Med Chem 2004; 11 : 369-87. 